\title{
Tendência secular de crescimento em estatura no município de Florianópolis (SC), Brasil, e sua associação com o índice de desenvolvimento humano (IDH)
}

\author{
Secular trend of growth in stature \\ in Florianópolis in the state of Santa Catarina (Brazil) \\ in relation with the human development index (HDI)
}

Andressa Caroline Carneiro Pinheiro ${ }^{1}$

Juliana Minuzzi Niederauer ${ }^{1}$

Deisi Maria Vargas ${ }^{1}$

${ }^{1}$ Departamento da Medicina, Programa de Pós-Graduação em Saúde Coletiva, Centro de Ciências da Saúde, Universidade Regional de Blumenau-Campus 1. R. Antônio da Veiga 140, Victor Konder. 89.012-900 Blumenau SC Brasil. deisivargas@furb.br
Abstract The article seks to evaluate the secular trend of growth in stature of recruits in the 63rd Infantry Battalion in Florianópolis and correlate the information with the human development index (HDI). It involves a transversal and retrospective study of recruits aged between 18 and 20 who joined the 63rd IB in Florianópolis from 1963 to 2007. The sample comprised 600 individuals out of a total of 3000 recruits enlisted over the period. In each decade, three years were selected and within these years the first 40 files were systematically selected for analysis. It was seen that there was an increase in the order of $7 \mathrm{~cm}$ in height of recruits in Florianopolis over the past 47 years. This increase was more marked between the decades of 1990 and 2000, with the municipality of Blumenau having the highest average. The average heights study over the decades showed a strong positive correlation with the HDI of Florianopolis during the same period. When comparing the heights of the capital of Santa Catarina and previous studies in Blumenau, it was found that both cities have achieved the same increase of $1.4 \mathrm{~cm} /$ decade in the period between the 1960 and 2000. There was a positive secular trend in growth in Florianopolis, with a strong correlation with HDI values of the city between 1960 and 2000.

Key words Growth, Stature, Military, Human development
Resumo O objetivo deste artigo é avaliar a tendência secular de crescimento (TSC) em estatura dos recrutas do $63^{\circ}$ Batalhão de Infantaria (BI) de Florianópolis (SC) e correlacioná-la com o indice de desenvolvimento humano (IDH). Estudo transversal e retrospectivo de recrutas com 18 a 20 anos que foram incorporados ao $63^{\circ} \mathrm{BI}$ de Florianópolis por alistamento militar no período de 1963 a 2007. A amostra foi constituida por 600 inscritos de um total de 3000 recrutas incorporados no período mencionado. De cada década, selecionou-se três anos e destes anos foram selecionadas sistematicamente as primeiras 40 fichas para análise. Constatou-se que em Florianópolis ocorreu acréscimo de $7 \mathrm{~cm}$ na estatura dos recrutas nos últimos 47 anos, sendo este incremento mais evidente entre as décadas de 1990 e 2000. As estaturas médias das décadas mostraram correlação forte e positiva com o IDH de Florianópolis. No comparativo das estaturas da capital catarinense e estudos anteriores realizados em Blumenau, encontrou-se para ambas as cidades o mesmo acréscimo de 1,4 cm/década no período entre as décadas de 1960 e 2000. Encontrou-se TSC positiva para Florianópolis, havendo forte correlação com os valores de IDH da cidade entre as décadas de 1960 e 2000.

Palavras-chave Crescimento, Estatura, Militares, Desenvolvimento humano 


\section{Introdução}

O acompanhamento do acréscimo em estatura pode ser considerado como meio de avaliação do padrão de vida das populações e como estimativa das consequências físicas de uma série de acontecimentos históricos, que incluem desde mudanças de hábitos alimentares até o impacto de conflitos militares. Tais alterações sociais podem ser demonstradas a partir de estudos baseados na resposta física de determinadas populações; possibilitando, entre outras análises, a eficiência das políticas públicas. O conceito de tendência secular de crescimento (TSC) foi utilizado primeiramente em 1829 pelo francês Villermé. De acordo com o autor: "[...] As circunstâncias que acompanham a pobreza atrasam a idade em que a estatura final é alcançada e diminuem a estatura adulta $[\ldots . .$. ".

A TSC baseia-se no encadeamento de fenômenos de ordem celular, fisiológica e morfológica. Tais fenômenos são pré-determinados geneticamente e potencialmente modificáveis pelo meio social, tendo em vista que apesar do componente genético ser a base para a estatura final, este não pode anular a influência ambiental ${ }^{1}$. Mesmo tornando-se difícil a quantificação exata da influência dos fatores genéticos e ambientais sobre o crescimento de uma população, a TSC em estatura permite a percepção dos fatores potencialmente modificáveis, ou seja, o componente ambiental e socioeconômico na determinação da estatura final ${ }^{2}$.

Os processos de crescimento em que o indivíduo está inserido não são estáticos; podendo ser positivos, negativos ou mesmo nulos. Sociedades que possuem tendência secular positiva quanto ao crescimento estatural demonstram superação dos fatores ambientais deficitários ou mesmo a melhora destes, resultando em acréscimo na altura final. Logo, a estatura humana alcança maiores níveis e o crescimento ocorre mais rapidamente na proporção em que sejam maiores as riquezas do país, melhores sejam as casas, as roupas e a nutrição; e menores sejam o trabalho, a fadiga e a privação durante a infância e a juventude ${ }^{3}$.

De uma maneira geral, as análises das curvas de tendência de crescimento permitem comparar o desenvolvimento social e econômico de diferentes países e localidades. Segundo dados da National Center for Health Statistics/Organização Mundial de Saúde (NCHS/OMS), ao redor dos 7 anos de vida os déficits médios de estatura da população brasileira, com relação ao padrão internacional de crescimento, ultrapassam $3,5 \mathrm{~cm}$ nas meninas e $4,0 \mathrm{~cm}$ para os meninos. A última pesquisa de curvas de crescimento realizada pela NCHS/OMS, em 2007, demonstrou que de acordo com dados coletados em Pelotas (RS), os brasileiros apresentavam prevalência de $2,5 \%$ de déficit de altura, valor esse similar aos 2,3\% esperados a partir da referência NCHS/OMS de crescimento de $1977^{4}$.

Assim como a TSC, o IDH é considerado atualmente uma importante alternativa no monitoramento de mudanças nos padrões socioeconômicos ${ }^{5}$. No Brasil, os valores de IDH foram da ordem de 0,394 em 1960, alcançando 0,757 em 2000 e 0,813 em 2007. Santa Catarina possui seis das dez regiões metropolitanas mais bem colocadas no ranking de IDH do país. Sua capital Florianópolis apresenta IDH semelhante aos países Europeus, sendo a cidade com o melhor IDH do Brasil seguida de Joinville e Blumenau (ambas com IDH de 0,85$)^{6}$.

Tendo em vista as condições socioeconômicas observadas no Brasil nas últimas décadas e suas repercussões, pretende-se neste trabalho caracterizar a tendência secular de crescimento em estatura no município de Florianópolis verificando se variações na estatura média apresentam correlação com as variações no IDH médio, além de confrontar os dados obtidos no estudo da capital catarinense com outro sobre tendência secular em crescimento na cidade de Blumenau (SC).

\section{Material e métodos}

Trata-se de um estudo transversal em recrutas com idades entre 18 e 20 anos que foram incorporados ao $63^{\circ}$ Batalhão de Infantaria de Florianópolis por ocasião do alistamento militar no período de 1963 a 2007. A significância da amostra e obtenção dos dados foram possíveis tendo em vista a obrigatoriedade do serviço militar no Brasil. No momento da apresentação à Junta Militar, registram-se traços fenotípicos (estatura, peso, cor da pele e do cabelo) e algumas características sócio demográficas (local de nascimento, escolaridade, profissão), dados que permanecem armazenadas em um arquivo com fichas separadas por ano de incorporação à instituição.

A amostra foi constituída por 600 recrutas de um total de 3000 incorporados no período, os quais foram uniformemente distribuídos nos anos pesquisados (margem de erro $<3,5 \%$ ). Foram consideradas elegíveis as fichas de alistamento de jovens com idade entre 18 e 20 anos, nascidos e procedentes da cidade de Florianópolis. 
Na coleta de dados, elegeu-se os 3 anos medianos de cada década, os terminados em 3, 5 e 7 com intuito de evitar a obtenção de dados de anos muito próximos porém de décadas diferentes. Foram estudadas de forma sistemática as primeiras 40 fichas de cada ano elegido. A antropometria dos recrutas foi realizada com antropômetro de metal (precisão $0,1 \mathrm{~cm}$ ) acoplado a balança mecânica (precisão $100 \mathrm{~g}$ ), com o indivíduo descalço e na presença de um médico.

Os resultados obtidos no presente trabalho foram então confrontados para discussão com os de estudo de TSC realizado na cidade de Blumenau.

\section{Análise Estatística}

Calculou-se as médias das estaturas dos anos estudados (anos finais 3, 5 e 7) e a média de cada década (anos finais 5). O teste ANOVA e o Teste de Turkey de comparações múltiplas foram utilizados na comparação das médias estaturais das décadas de 1960, 1970, 1980, 1990 e 2000. Para o calculo do $Z$-score da estatura utilizou-se os valores de referência do Center for Desease Control $(\mathrm{CDC})^{7}$. Estes valores de referência foram confrontados com a média de cada década (1960, 1970, 1980, 1990 e 2000).Na análise do IDH utilizou-se os valores do IDH médio dos anos de $1970,1980,1990$ e 2000 . Estes anos foram correlacionados com as estatura médias dos anos próximos 1973, 1983, 1993 e 2003 através da equação de regressão linear simples. Considerou-se nível de significância estatística valores de $\mathrm{p} \leq$ 0,05. Este trabalho foi aprovado pelo Comitê de Ética da Universidade Regional de Blumenau.

\section{Resultados}

Encontrou-se acréscimo de $7 \mathrm{~cm}$ na estatura média final dos recrutas do $63^{\circ}$ Batalhão de Infantaria de Florianópolis (SC) no período de 1963 a 2007. O acréscimo em estatura não ocorreu de forma progressiva, sendo mais acentuado no intervalo entre as décadas de 1990 e 2000 . Neste período foi observado um aumento da ordem de $3 \mathrm{~cm}$ em relação à década anterior. Esta variação foi quase três vezes maior que a média de $1,16 \mathrm{~cm}$ de incremento por década encontrado nos anos antecedentes. Encontraram-se diferenças significantes entre as médias estaturais das décadas analisadas (Tabela 1). Através da equação de regressão linear simples constatou-se uma correlação positiva e forte $(r=0,95$ e $p \leq 0,05)$ entre a estatura dos recrutas e os anos analisados (Gráfico 1).

Em relação aos valores de referência do CDC observou-se aumento estatural progressivo. A estatura média da década de 60 correspondeu a um Z-score de -1,04 passando para -0,89 DP na década de 1970, - 0,28 DP nas décadas de 1980 e 1990 e - 0,2 DP na década de 2000.

Observou-se um aumento progressivo do IDH de Florianópolis e do Brasil no período estudado (Tabela 2). A regressão linear simples mostrou uma correlação positiva e forte entre as estaturas e o IDH médio da cidade de Florianópolis $(\mathrm{r}=0,951 ; \mathrm{p}<0,001)$. (Gráfico 2).

No comparativo dos dados da média estatural entre Florianópolis e Blumenau e os anos pesquisados, observou-se que as duas cidades obtinham a mesma estatura média no início do estudo em 1963. Durante o período estudado, as duas cidades alcançaram o mesmo ganho estatural final de $7 \mathrm{~cm}$; sendo que tal incremento mostrouse diferente durante os anos estudados para as duas cidades. Florianópolis obteve seu maior ganho em estatura em entre as décadas 1990 e 2000 e Blumenau entre 1970 e 1980.

\section{Discussão}

O acréscimo observado na tendência secular de crescimento em estatura na cidade de Florianó-

Tabela 1. Distribuição das médias das alturas em metros (m) por ano e década.

\begin{tabular}{ccc}
\hline Ano & $\begin{array}{c}\text { Altura do ano } \\
(\text { metros) }\end{array}$ & $\begin{array}{c}\text { Altura da } \\
\text { década }^{*} \text { (metros) }\end{array}$ \\
\hline 1963 & 1,68 & \\
1965 & 1,68 & \\
1967 & 1,69 & \\
1973 & 1,69 & 1,70 \\
1975 & 1,70 & \\
1977 & 1,70 & \\
1983 & 1,70 & \\
1985 & 1,70 & \\
1987 & 1,71 & 1,72 \\
1993 & 1,72 & \\
1995 & 1,72 & \\
1997 & 1,72 & \\
2003 & 1,75 & \\
2005 & 1,75 & \\
2007 & 1,75 & \\
\hline
\end{tabular}

"ANOVA $\mathrm{p}<0,0001$; Turkey $\mathrm{p}<0,05$ entre 1965 versus 1995 , 1965 versus 2005; 1975 versus 2005 e 1985 versus 2005. 


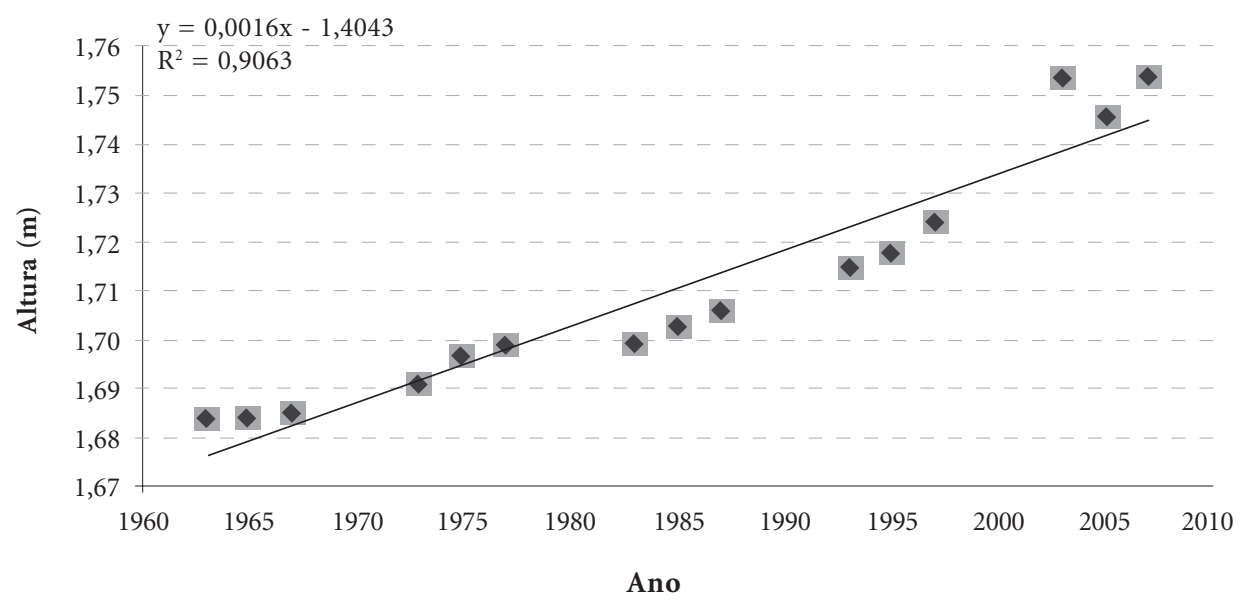

Gráfico 1. Gráfico de regressão linear simples correlacionando as médias em estatura da cidade de Florianópolis e os anos pesquisados.

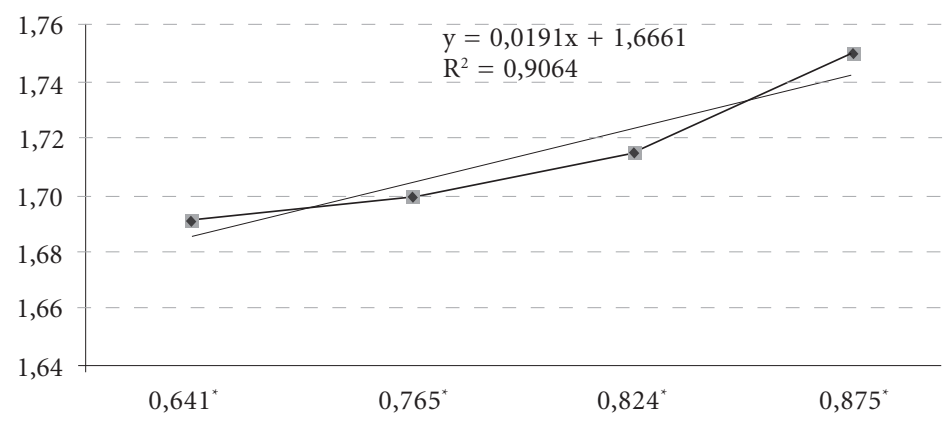

Gráfico 2. Gráfico de regressão linear simples correlacionando as médias em estatura dos anos 1973 - 1983 1993 e 2003 com o IDH dos anos próximos* (1970 - 1980 - 1990 - 2000) da cidade de Florianópolis.

*Fonte: SEBRAE-SC, 2010.

Tabela 2. Distribuição das estaturas médias e IDH médio* por ano.

\begin{tabular}{cccc}
\hline Ano & $\begin{array}{c}\text { Estatura } \\
\text { (metros) }\end{array}$ & $\begin{array}{c}\text { IDH }^{* *} \\
\text { Florianópolis }\end{array}$ & $\begin{array}{c}\text { IDH }^{\#} \\
\text { Brasil }\end{array}$ \\
\hline 1973 & 1,69 & 0,641 & 0,462 \\
1983 & 1,70 & 0,765 & 0,695 \\
1993 & 1,72 & 0,824 & 0,742 \\
2003 & 1,75 & 0,875 & 0,752 \\
\hline
\end{tabular}

${ }^{*}$ Dos anos 1970,1980,1990 e 2000. ${ }^{* *}$ Fonte: SEBRAE-SC, 2005.

${ }^{*}$ Fonte: UNDP, Human development report, 1998. polis foi significativamente positivo e demonstrou que a população está superando as condições adversas do meio ambiente e desta maneira aproximando-se da estatura que seria proporcionada pela plenitude do potencial genético. $\mathrm{O}$ acréscimo em estatura de uma população pode ser entendido como uma variável intimamente relacionada ao progresso socioeconômico e à melhoria dos meios de subsistência ${ }^{8}$. Sendo assim, os valores positivos de TSC em Florianópolis podem ser considerados evidências biológicas 
do registro cumulativo do histórico nutricional, bem como da melhora das condições de vida da população e das políticas socioculturais e econômicas governamentais.

Em estudos sobre TSC em estatura e índice de massa corporal (IMC) nas crianças chinesas, encontraram-se acréscimos de estatura na faixa de $11,8 \mathrm{~cm}$ para os meninos e $11,1 \mathrm{~cm}$ para as meninas no comparativo das crianças chinesas em 2005 para a mesma geração 50 anos atrás. Segundo os autores, os resultados positivos para TSC e IMC permitiram a constatação do papel benéfico das políticas públicas e poderia inclusive predizer positivamente sobre o desenvolvimento da sociedade e de seus marcadores sociais e econômicos ${ }^{9}$.

Em relação às crianças japonesas, foram constatadas mudanças na tendência secular de crescimento associadas a alterações provocadas pela segunda guerra mundial. No período pré-conflito, até 1939 , foi constatada tendência secular positiva em estatura, tendo este parâmetro entrado em declínio a partir de 1939 quando o Japão entrou na guerra. Houve um pico de declínio no final da guerra e nos dois anos seguintes. Estes anos foram marcados por fome e destruição devido à desorganização econômica ocorrida pela derrota sofrida pelo país. Interessante notar que após este período de pós-guerra houve um rápido crescimento e desenvolvimento do país, ocasionando uma tendência secular positiva nos sete anos seguintes quando as crianças finalmente atingiram a estatura do período pré-conflito ${ }^{10}$. Outra questão relacionada à influência da segunda grande guerra no crescimento em estatura dos jovens japoneses foi levantada quanto à mudança dos hábitos alimentares. Encontraram-se grandes mudanças na alimentação no período pós-guerra devido a ocidentalização dos hábitos e costumes além da variação na nutrição rica em cereais, principalmente arroz, pobre em cálcio; para uma dieta rica em carnes, leite e outras fontes de proteína. Sabendo que os ossos longos são responsáveis pela estatura e o crescimento e estes dependem da ingestão de cálcio, os autores da pesquisa relacionaram o aumento da estatura de jovens japoneses no período de 1920 e 1960 com o aumento da ingestão de leite e demais proteínas ${ }^{11}$.

No Brasil, foi encontrada queda das médias estaturais durante o período pós-guerra mundial devido à conjuntura econômica mundial desfavorável, que acabou por influenciar a economia brasileira da época apesar da discreta participação do país nos conflitos. A inflação que havia sido de apenas 39\% entre 1929 e 1939, elevou-se para 129\% no período de guerra, tendo decaído para 86\% entre 1940 e 1947. Outra informação relevante refere-se à alta dos preços de 19 gêneros alimentícios básicos, que havia sido de 11\% ao ano entre 1935 e 1938, tendo disparado para 103\% entre dezembro de 1940 e novembro de $1944^{12}$.

Além das TSC positivas e negativas, há nações que experimentam valores nulos. Este é o caso da Polônia, onde pesquisas realizadas apontaram uma TSC nula possivelmente devido ao alcance do total potencial genético possível para estatura da população, ou mesmo porque as condições socioeconômicas do país cessaram seu crescimento. Os autores basearam-se em dados obtidos de 1880 até o ano 2000 e concluíram que até 1980 houve aceleração do desenvolvimento físico das crianças polonesas, a partir desta data os dados indicaram uma redução ou mesmo o desaparecimento da aceleração no crescimento vista até então ${ }^{13}$.

Dentre as alternativas no monitoramente das mudanças dos padrões econômicos de saúde e nutrição, cita-se além da TSC o índice de desenvolvimento humano. A relação entre os indicadores econômicos ocorre em virtude da compatibilidade das TSC com indicadores de saúde, posto que alterações nos padrões de crescimento refletem mudanças de mortalidade e morbidade ${ }^{14}$. Acrescenta-se ao fato, ser a relação da altura para idade um ponto com significância preditiva do nível de desenvolvimento cognitivo e desempenho escolar ${ }^{15}$. Além disso, foi demonstrado que a redução de 0,1 no coeficiente de Gini (parâmetro internacional usado para medir a desigualdade de distribuição de renda entre os países) poderia resultar em uma redução de valores próximos a $3,0 \mathrm{~cm}$ na estatura final de uma população ${ }^{16}$.

Os dados obtidos em Florianópolis para a TSC demonstram que durante 1963-2007 houve acréscimo de $7 \mathrm{~cm}$ ou $0,14 \mathrm{~cm} /$ década na estatura final dos recrutas. Este valor de TSC mostrou-se semelhante a outros estudos realizados no $\mathrm{Sul}$ do Brasil. Em Blumenau (SC), observou-se a mesma média de acréscimo por ano $0,14 \mathrm{~cm} /$ década entre 1963 e 2007 e associação positiva entre a evolução da estatura e a variação do IDH $^{17}$. $\mathrm{Na}$ análise da TSC em estatura dos recrutas, realizada em cidades do sul do Brasil no período compreendido entre 1940 e 1969 foram encontradas os seguintes valores: Rio Grande (RS) $(0,15 \mathrm{~cm} /$ ano $)$, Pelotas (RS) $(0,10 \mathrm{~cm} /$ ano $)$ e Bagé (RS) $(0,08 \mathrm{~cm} / \mathrm{ano})^{18}$.

No comparativo entre as cidades de Florianópolis e Blumenau, a TSC foi positiva para ambas com um aumento estatural de $7 \mathrm{~cm}$ para 
o mesmo período. Apesar do incremento estatural ter sido igual em um mesmo período de tempo estudado, as estaturas médias por década foram diferentes assim como sua progressão. Em Florianópolis os recrutas apresentaram na década de 1960 uma média estatural de 1,69 metros versus 1,70 metros em Blumenau, e na década de 2000 uma média estatural de 1,75 metros versus $1,77 \mathrm{~cm}$. O maior incremento em estatura na cidade de Blumenau ocorreu entre as décadas de 1970 e 1980 alcançando o valor de 4 cm enquanto a cidade de Florianópolis atingiu o ápice de incremento estatural entre as décadas 1990 e 2000 quando foi constatado um acréscimo de $3 \mathrm{~cm}$.

O pico de acréscimo estatural em Florianópolis está em concordância com uma importante redução documentada a partir de inquéritos probabilísticos de abrangência nacional realizados em um intervalo entre 1996 e 2006. Neste período foi encontrado descréscimo importante de aproximadamente $50 \%$ na prevalência da desnutrição infantil no Brasil. Tal alteração foi considerada como consequência da melhoria das condições de saúde e saneamento, bem como o poder aquisitivo das famílias e escolaridade das mães ${ }^{19}$.

O maior acréscimo em estatura apresentado por Blumenau apresenta similaridade temporal com a significativa expansão do Programa de Saúde da Família (PSF) do Governo Federal na década de oitenta, o qual possibilitou melhora substancial do acesso de mães e crianças à assistência saúde ${ }^{19}$.

Além dos fatores socioeconômicos, as diferenças nas estaturas médias podem estar relacionadas a fatores genéticos já que diferentes grupos populacionais foram predominantes na colonização destas cidades. Houve predominância de açorianos em Florianópolis e germâmicos em Blumenau ${ }^{20}$. Apesar do maior aumento de IDH em ambas as cidades ter ocorrido no mesmo período, entre as décadas de 70 e 80 , o maior incremento estatural não ocorreu de forma paralela como exposto no paragrafo anterior. Considerando a classificação do IDH em baixo = entre 0 e 0,$499 ;$ médio $=$ entre 0,500 e 0,799 e alto $=$ entre 0,800 e 1,0; foi possível constatar que em Blumenau, o IDH no início a década de 1980 era de 0,797 , muito próximo à classificação de um IDH alto, enquanto em Florianópolis o IDH era de 0,765 . É possível que as variações temporais dos valores do IDH destas cidades possam ter refletido na magnitude dos acréscimos estaturais alcançadas nas diferentes décadas em cada uma delas, justificando o fato de Blumenau e Florianópolis terem apresentado maior aumento estatural em décadas diferentes.

Os acréscimos importantes encontrados nas cidades de Blumenau e Florianópolis demonstraram a posição privilegiada de Santa Catarina quanto ao desenvolvimento humano e elevação do nível de renda, num ritmo muito superior à média regional. Contudo, este incremento não é homogêneo quando compara-se cidades do Vale do Itajaí e capital catarinense, com as cidades localizadas na região central e noroeste do Estado ${ }^{21,22}$.

Estes achados reforçam o indicativo de que quanto melhor forem os valores de expectativa de vida, renda per capita e educação (componentes do IDH), menores serão os fatores que encerram redução da estatura final e maiores são as possibilidades dos indivíduos alcançarem seu potencial genético. Neste intuito, para a perpetuação e melhora dos marcadores IDH e consequentemente dos marcadores biológicos, tornase importante assegurar políticas públicas que propiciem acesso amplo da população brasileira a serviços básicos, tais como a saúde e educação.

\section{Conclusão}

Observou-se uma TSC em estatura positiva na cidade de Florianópolis da ordem de 1,4 cm/década ou 0,14 cm/ano entre os anos de 1963 e 2007 que associou-se positivamente e com o IDH. No comparativo com Blumenau encontrou-se que as duas cidades obtiveram o mesmo acréscimo estatural, embora este tenha ocorrido de maneira disforme no decorrer dos anos para as duas cidades. 


\section{Colaboradores}

ACC Pinheiro, JM Niederauer e DM Vargas participaram igualmente de todas as etapas de elaboração do artigo.

\section{Referências}

1. Silva Neto LG. Crescimento, composição corporal e performance motora em crianças e adolescentes de 07 a 14 anos provenientes de famílias de baixo nível sócio-econômico e participantes do projeto esporte solidário, São Luis-MA [dissertação]. Campinas: Universidade Estadual de Campinas; 1999.

2. Malina RB. Crescimento de crianças latino americanas: comparação entre os aspectos sócio-econômicos, urbano-rural, e tendência secular. Revista Brasileira de Ciência e Movimento 1990; 4(3):46-75.

3. Monteiro CA, Conde WL. Secular trends in postnatalgrowth in S. Paulo city, Brazil (1974-1996). Rev Saude Publica 2000; 34(6):41-51.

4. Victora CG, Araújo CL, de Onis M. Uma nova curva de crescimento para o século XXI. Rev. paul. pediatr. 2010; 28(4):1-20.

5. Panhoca da Silva OM, Panhoca L. A contribuição da vulnerabilidade na determinação do índice de desenvolvimento humano: estudando o estado de Santa Catarina. Cien Saude Colet 2007; 12(5):1209-1219.

6. Serviço Brasileiro de Apoio às Micro e Pequenas Empresas. (Sebrae). Santa Catarina em números. Florianópolis: Sebrae; 2010. [página na Internet]. [acessado $2011 \mathrm{fev} 11$ ]. Disponível em: http://www. sebrae-sc.com.br/scemnumero

7. U.S. Department of Health and Human Services. Centers for Disease Control and Prevention (CDC). National Center for Health Statistics (NCHS). [página na Internet]. [acessado 2011 jan 11]. Disponível em: http://www.cdc.gov/growthcharts

8. Bogin B, Keep R. Eight thousand years of economic and political history in Latin America revealed by anthropometry. Annals of human biology 1999; 26(4):333-351.

9. Cheng-Ye J, Tan-Jian C. Secular Changes in Stature and Body Mass Index for Chinese Youth in Sixteen Major Cities, 1950s-2005. Am J Hum Biol 2008: 20(5):530-537.

10. Takahshi E. Growth and environmental factors in Japan. Human Biology 1966; 38:112-130

11. Murata M, Hibi I. Nutrition and the secular trend of growth. Horm Res 1992; 38(1):89-96.

12. Oliveira A, Lobo EML. O Estado novo e o sindicato corporativista, 1937-1945. Rio de Janeiro Operário: Natureza do Estado, a Conjuntura Econômica, Condições de Vida e Consciência de Classe, 19301937. 1992; 102-127.
13. Krawczynski M, Walkowiak J. Secular changes in body height and weight in children and adolescents in Poznan, Poland, between 1880 and 2000. Acta Pediatr 2003; 92(3):277-282.

14. Van Wieringen JC. Secular growth changes. In: Human Growth. A Comprehensive Treatise. Methodology, Ecological, Genetic and Nutritional Effects on Growth 1986; 307-331.

15. Simeon D, Grantham-McGregor S. M. Nutritional deficiencies and children's behavior and mental development. Nutrition Research Reviews 1990; 3(1):1-24.

16. Steackel RH. Heightand per capita income. Historical Method 1983; (16):1-7.

17. Vargas DM, Soncini AS, Arena MGL, Arena LFGL. Tendência secular de crescimento em Blumenau Brasil e a sua associação com o índice de desenvolvimento humano (IDH). Rev Assoc Med Bras 2010; 56(3):304-308.

18. Kac G. Tendência secular em estatura: uma revisão da literatura. Cad Saude Publica 1999; 15(3):451461.

19. Monteiro CA, Benicio MH, Konno SC, Silva ACF, Lima ALL, Conde WL. Causas do declínio da desnutrição infantil no Brasil, 1996-2007. Rev Saude Publica 2009; 43(1):35-43.

20. Piazza WF. A Colonização de Santa Catarina. Florianópolis: Lunardelli; 1994.

21. Panhoca L, Silva OMP. A contribuição da vulnerabilidade na determinação do índice de desenvolvimento humano: estudando o estado de Santa Catarina. Cien Saude Colet 2007; 12(5):1209-1219.

22. Lavinas L, Garcia EH, Amaral MR. Desigualdades Regionais: Indicadores Socioeconômicos nos Anos 90. Rio de Janeiro: Instituto de pesquisa econômica aplicada (IPEA); 1997. (Texto para Discussão, No 460)

Artigo apresentado em 17/10/2012

Aprovado em 26/01/2013

Versão final apresentada em 04/02/2013 
